Departamento de Historia Universidad de Santiago de Chile

Revista de Historia Social

y de las Mentalidades

Volumen 24, N², 2020: 97-120

Issn Online: 0719-4749

\title{
FRANQUISMO E IBEROAMÉRICA A TRAVÉS DE LOS CONVENIOS DE SEGURIDAD SOCIAL, 1959-1974. UNA APROXIMACIÓN DESDE ESPAÑA*
}

\author{
FRANCOISM AND IBERO-AMERICA THROUGH SOCIAL SECURITY AGREEMENTS, 1959- \\ 1974. AN APPROACH FROM SPAIN
}

\author{
MG. GUILLERMO REVUELTA SIERRA** \\ Universidad de Cantabria \\ Santander, España \\ Email: guillermo.revuelta.sie@gmail.com \\ Id-Orcid: 0000-0003-4052-4960
}

\begin{abstract}
RESUMEN
Desde los años sesenta hasta la primera mitad de los setenta del siglo XX, la emigración española se disparó a países europeos, mientras se reducía en los países iberoamericanos. En este momento, empero, se firmaron convenios de seguridad social entre el franquismo y los Estados iberoamericanos que establecieron nuevas formas de relación entre

ambas partes. Nuestro propósito es explicar los motivos por los que se firmaron y analizar sus prestaciones. Como novedad más reseñable, este trabajo estudia la documentación del Boletín Oficial de las Cortes Españolas (BOCE), ignorada prácticamente por la historiografía.

Palabras clave: Convenios; seguridad social; franquismo; Iberoamérica
\end{abstract}

\begin{abstract}
From the 1960s to the early 1970s of 20th century, Spanish emigration shot up to European countries, while it was reduced in Ibero-American countries. At this time, however, agreements about social security were signed between francoism and the Ibero-America states that established new forms of relationship between the two parties. Our purpose is to explain the reasons why they were signed and to analyze their benefits. As a more remarkable novelty, this work studies the documentation of the Boletín Oficial de las Cortes Españolas (BOCE), practically ignored by historiography.
\end{abstract}

Keywords: Agreements; Social security; Francoism; Ibero-America

* $\quad$ Recibido: 6 de mayo de 2020; Aprobado: 30 de junio de 2020.

** Artículo Científico. Este trabajo se enmarca dentro de la investigación doctoral "La previsión social del régimen de Franco", con el apoyo del programa "Concepción Arenal" de la Universidad de Cantabria. 
Cómo citar: Revuelta Sierra, Guillermo. (2020). "Franquismo e Iberoamérica a través de los convenios de seguridad social. Una aproximación desde España”. Revista Historia Social y de las Mentalidades, 24(2), 97-120. DOI: $10.35588 /$ rhsm.v24i2.4453

\section{INTRODUCCIÓN}

En este texto estudiaremos los convenios sobre seguridad social firmados por el régimen franquista y diversos Estados iberoamericanos de Argentina, Brasil, Ecuador, Paraguay y Perú. El análisis lo realizaremos en dos sentidos, que tendrán su correspondencia en los dos grandes apartados del trabajo. En primer lugar, mostraremos la doctrina española respecto al tema; las relaciones que establecieron los países iberoamericanos referidas a la seguridad social durante la década de los cuarenta, y la intromisión del régimen franquista en tales relaciones, tomando una posición rectora con la Organización Iberoamericana de Seguridad Social (OISS) a lo largo de los años cincuenta. Terminaremos mostrando los factores socioeconómicos que se dieron a partir de los sesenta.

En segundo lugar, analizaremos los convenios. No pretendemos un estudio jurídico o excesivamente pormenorizado de los convenios, sino revisar aspectos concretos: qué legislaciones se aplicarían, a qué personas se dirigían, y el modo en que los trabajadores obtendrían las prestaciones, así como cuáles eran las instituciones competentes de garantizarlas. Estos aspectos nos permitirán conocer cómo los firmantes de los acuerdos, es decir, los Estados, organizaron sus sistemas de seguridad social, y qué compromisos adquirían con los inmigrantes. Antes de cada análisis plantearemos, someramente, nuestras hipótesis con relación a por qué los políticos iberoamericanos se vieron interesados en firmar los convenios con España y por qué se acordaron en tales fechas, salvo en el caso paraguayo, en que la bibliografía general aporta escasas informaciones. El apartado se estructurará en dos subapartados debido a los tipos de convenios suscritos. Finalizaremos el estudio con unas conclusiones abiertas, dado el carácter aproximativo del mismo.

La documentación que emplearemos es la del Boletín Oficial de las Cortes Españolas (BOCE), que se encuentra digitalizada en la página web del Congreso de los Diputados. ${ }^{1}$ El valor del estudio del BOCE reside en que no todos los acuerdos adoptados entre el franquismo y los países iberoamericanos llegaron a ratificarse de manera que no aparecen publicados en el Boletín Oficial

1 La documentación se encuentra digitalizada en congreso.es/est_sesiones/ 
del Estado (BOE), pero sí pasaron por las Cortes, lugar donde se encuentran tales acuerdos como es el caso del convenio hispano-colombiano.

Hay que indicar, también, que, a pesar de que sería muy interesante integrar los efectos que estos acuerdos tuvieron sobre la realidad social de los trabajadores emigrantes y sus familias, no existen estudios historiográficos que hayan abordado el tema. Esta investigación, al observar el marco normativo, pretende suplir en parte este vacío, estudiando el marco institucional con el cual los Estados se comprometían a intervenir en la realidad social de los migrantes. Por ello, en el estudio de los convenios analizaremos con exhaustividad la protección que los gobiernos firmantes se comprometían a otorgar a los inmigrantes, así como los modos de proporcionarla. Por supuesto, somos conscientes de que este compromiso no tuvo por qué materializarse, pero cualquier estudio que quiera conocer la realidad social y las percepciones de los migrantes sobre la seguridad social, necesita del conocimiento de los compromisos institucionales reflejados en la normativa. Y esto es así porque la seguridad social es un servicio ofertado y garantizado por los Estados, aunque las formas de hacerlo sean diversas.

\section{PRECEDENTES Y CONDICIONANTES POLÍTICOS Y SOCIOECONÓMICOS}

Desde 1959, el régimen de Franco firmó una serie de convenios sobre seguridad social con países iberoamericanos, constituyéndose en una nueva forma de relación en el tema entre los Estados iberoamericanos y el español. El establecimiento de estos convenios supuso una ruptura con lo sostenido por la doctrina española hasta el momento, en torno a una equiparación absoluta de los inmigrantes iberoamericanos, andorranos, y filipinos con los trabajadores nacionales; y la "reciprocidad diplomática" con los no iberoamericanos. La excepción a esta diferenciación se daba en el seguro de accidentes de trabajo, ya que la equiparación era absoluta entre los trabajadores accidentados, fuesen españoles e iberoamericanos o no iberoamericanos, aunque se distinguía entre los derecho-habientes, en que, de nuevo, los no iberoamericanos quedaban ligados a los criterios de reciprocidad y residencia (Borrajo/Alonso 57-58/225-228). Por el contrario, no suponía una ruptura con la experiencia de relaciones previas, de las cuales, ciertamente, estas firmas eran una consecuencia.

Desde la década de los treinta, en los países iberoamericanos se comenzaron a establecer instituciones estatales para organizar y supervisar los sistemas de seguridad social (Álvarez 20). Pronto, con el apoyo de la Oficina Internacional del Trabajo (OIT), se concretaron acuerdos intergubernamentales y se crearon instituciones regionales para el desarrollo de los sistemas de seguridad social. 
En 1938, se constituyó el Comité Interamericano de Iniciativas en Materia de Seguridad Social a fin de que los Gobiernos e instituciones dedicadas a la materia tuviesen un organismo permanente de estudio, colaboración, información y acción teórica. Como resultado, el Comité celebró entre el 10 y 16 de septiembre de 1942 en Chile, la primera Conferencia Iberoamericana de Seguridad Social, a la que asistieron representantes de veintiún países: Argentina, Bolivia, Brasil, Canadá, Colombia, Costa Rica, Cuba, Chile, Ecuador, El Salvador, EE.UU., Guatemala, Haití, México, Nicaragua, Panamá, Paraguay, Perú, República Dominicana, y Uruguay, así como representantes de la OIT, la Oficina Panamericana y el Instituto Internacional Americano de Protección a la Infancia (Vilar y Pons 3-4). ${ }^{2}$ Se celebraron dos conferencias más, en Brasil en 1947 y en Argentina en 1951, que no atrajeron demasiada atención, pero que igualmente siguieron siendo relevantes para la difusión de reformas en los sistemas de seguridad social en todo el continente (Álvarez 21).

Los dirigentes franquistas eran conscientes de esta realidad. Junto a la necesidad de reforzar el papel de España en el concierto internacional, los franquistas se veían motivados a participar en este entramado regional por dos motivos: creían en la ejemplaridad del sistema de seguridad social español para los países iberoamericanos, y más teniendo en cuenta las raíces culturales comunes; y pensaban que la participación de EE.UU. como miembro en el Comité, significaba la presencia de una posición teórica diferente a la del franquismo. Por ello, los Ministros de Asuntos Exteriores, Alberto Martín Artajo, y de Trabajo, José Antonio Girón de Velasco, así como el Instituto de Cultura Hispánica, se esforzaron en integrar España en tales circuitos. Las labores cristalizaron en el Primer Congreso Iberoamericano de Seguridad Social, celebrado en Madrid el año 1951, en la sede del Instituto de Cultura Hispánica. A este Congreso enviaron representantes Argentina, Bolivia, Brasil, Colombia, Costa Rica, Cuba, Chile, Ecuador, España, Filipinas, Haití, Paraguay, Perú, Portugal, República Dominicana, El Salvador, Uruguay, Venezuela, así como observadores de Italia, Bélgica, la Asociación Internacional de Seguridad Social y del Comité Iberoamericano de Seguridad Social, y se contó con el respaldo explícito de la OIT y de la Conferencia de Estados Americanos. A pesar del apoyo y la representación de organizaciones internacionales técnicas en el Congreso, el evento tuvo un evidente carácter político, marcado por la ausencia de países como EE.UU. y México. Lo más destacado de este Congreso fue la consolidación del Consejo Asesor de la Oficina Iberoamericana de Seguridad Social, que cumplía

2 Arturo Álvarez indica, sin embargo, que el Comité se estableció en Lima en 1940 (Álvarez 21). 
tres funciones: organizar seminarios de estudios sobre terminología, legislación comparada y problemas de aplicación práctica de la Seguridad Social; convocar becas y cursos de formación destinados a iberoamericanos para que conociesen el sistema de previsión social español; y editar publicaciones formativas y técnicas (Vilar y Pons 4). En el Segundo Congreso Iberoamericano de seguridad social en Lima desarrollado durante el año 1954, se aprobó la "Carta Constitucional de la OISS" (Vilar y Pons 5).

En noviembre de 1955, Martín Artajo propuso la creación de una "Comisión Interministerial de Convenios de Seguridad Social", que se materializó a través de un Decreto publicado en el BOE, en diciembre del mismo año. De hecho, pese a que la seguridad social del franquismo estuvo ligada orgánicamente al Ministerio de Trabajo, la Comisión quedaba integrada en el Ministerio de Asuntos Exteriores. Así, los cargos de Presidente, Vicepresidente y Secretario de tal comisión correspondían a quienes ocupasen los puestos rectores en subsecretarías y direcciones generales de este Ministerio (BOE, no. 335 7255). ${ }^{3}$ Ello pone de manifiesto que no era una necesidad socioeconómica, sino una elección política la que motivó el acuerdo de tales convenios. De este modo, la consecuencia práctica de esta elección en el ámbito tratado se dio en el Tercer Congreso Iberoamericano, celebrado en Quito en 1958, donde se acordó una extensión recíproca de la seguridad social en favor del afiliado de un determinado país, que se trasladase a un país iberoamericano. Se estableció la asistencia técnica y de servicios entre las instituciones de seguridad social de Iberoamérica y el reconocimiento recíproco de los derechos sociales de los países que suscribiesen el Tratado de Cooperación (Vilar y Pons 5).

Sin embargo, a pesar de todo este entramado de relaciones y acuerdos sobre seguridad social, la materialización de convenios sobre seguridad social fue mínima, pues solo se establecieron con cinco países distintos: Paraguay, Ecuador, Perú, Argentina y Brasil (Libro 383). Esto tiene que ver, sostenemos, con un cambio en las prioridades internacionales del régimen franquista tras la aplicación del Plan de Estabilización y Liberalización de 1959. Integrada España en el concierto internacional gracias a EE.UU., y con una creciente emigración a los países de Europa central y del norte, los dirigentes franquistas centraron sus esfuerzos en conseguir protección social para los españoles emigrados en tales países, lo que produjo que la política iberoamericana pasara a un segundo plano (Vilar y Pons 11). Esta prioridad se reforzó por el papel que adquirió la 
emigración europea en la planificación económica del régimen: primero, servía para contener el desempleo; y segundo, era una fuente de divisas, pues las remesas enviadas por los emigrantes, junto al turismo y las entradas de capital extranjero, permitieron cubrir el déficit tradicional de la balanza comercial española (Barciela et al. 298). En este contexto, la emigración de mano de obra española a Iberoamérica se tornó poco rentable para el régimen a causa de tres razones: Iberoamérica había vivido un fuerte crecimiento demográfico tras la Segunda Guerra Mundial, por lo que disponía de abundante mano de obra poco cualificada; la caída de las exportaciones de esta región provocó una fuerte crisis económica; y los países incentivaron la inmigración de trabajadores cualificados, favoreciendo la reagrupación familiar (Fernández y Kreienbrink 231).

Al régimen franquista no le convenía atender la demanda migratoria que estas condiciones socioeconómicas generaban. En primer lugar, la emigración iberoamericana era más espontánea que la europea, lo que dificultaba dirigirla y controlarla burocráticamente. En segundo lugar, la emigración iberoamericana afectaba fundamentalmente a regiones de la fachada atlántica, especialmente a Galicia. Al régimen, sin embargo, le convenía la emigración desde regiones como Andalucía y Extremadura, donde existía una alta concentración de paro y subempleo. La emigración, tal como la concebían las autoridades franquistas, serviría como "válvula de escape", evitando las tensiones y conflictos sociales que un alto porcentaje de parados y subempleados podría generar. La emigración europea, que estaba más distribuida entre todas las regiones de España, afectaba de forma importante a las dos regiones señaladas. En tercer lugar, la mano de obra demandada por los países iberoamericanos era la cualificada. Precisamente, esta era la mano de obra que el franquismo quería retener, pues resultaba esencial para el giro económico que había dado el régimen tras el Plan de Estabilización de 1959 y el establecimiento de Planes de Desarrollo en la década de los sesenta. La emigración europea no exigía tal mano de obra. Finalmente, en cuarto lugar, la emigración europea era, como la caracterizaban las autoridades del régimen, "indefinida". Esto significaba realmente que era definitiva. Eso querían los países iberoamericanos que potenciaron, en lo posible, la reagrupación familiar (algo que se verá en alguna de las cláusulas de los convenios). Este carácter derivaba en la reducción, cuando no paralización, del envío de remesas económicas (uno de los objetivos prioritarios de la política de emigración franquista y considerada esencial para su política económica). La emigración europea, por el contrario, solía ser individual, masculina, y temporal, lo que facilitaba el envío de las valiosas remesas, ya que los trabajadores dejaban en España a sus familias (Fernández y Kreienbrink 232-233). 


\section{LOS CONVENIOS SOBRE SEGURIDAD SOCIAL ENTRE ESPAÑA Y PAÍSES IBEROAMERICANOS}

A la hora de analizar los distintos convenios, encontramos dos tipos muy definidos, con diferencias claras en su estructura. Además, un tipo corresponde a la primera mitad de la década de los sesenta y el segundo al resto de esta década y la primera mitad de los setenta.

\subsection{Convenios de la primera época, 1959-1964}

Los Convenios del primer tipo fueron acordados con Paraguay (1959), ${ }^{4}$ Ecuador (1960), ${ }^{5}$ y Perú (1964). ${ }^{6}$ Además, hemos hallado la tramitación de un Convenio con Colombia en 1962, ${ }^{7}$ que no fue ratificado. Estos convenios constaban de cinco artículos (en el caso peruano de siete) y en todos los casos, salvo el de Paraguay, existía una breve exposición de motivos, que se resume en "el deseo de consagrar el principio de equiparación de trato" y "la conservación de los derechos adquiridos [...] cuando se trasladen" (BOCE, no. 669/no. 740 13975/15574-15575) para los trabajadores de los países contratantes. Salvo algunas diferencias en el convenio hispano-peruano, el resto de convenios eran idénticos en la redacción.

Los tres primeros artículos hacen referencia a la asistencia sanitaria. El primero garantizaba una asistencia médica y de maternidad inmediatas al trabajador emigrante al afiliarse a la Institución del otro país contratante, cuando tuviese reconocido ese derecho en la institución de seguridad social en su país de origen. El segundo artículo garantizaba la asistencia sanitaria "en caso de urgencia" a las personas en situación de afiliado en la institución de su país de origen y que viajasen al otro país "por diversos motivos", sin necesidad de estar afiliados a la institución de tal país, siempre que fuese "accidentalmente" y "toda vez que puedan justificar que están en uso de sus derechos en su institución respectiva" (BOCE, no. 641/ no. 669/ no. 740 13332/13976/15575). El cumplimiento del tercer artículo quedaba condicionado, por el propio convenio, al grado de desarrollo del sistema de seguridad social de los países contratantes, pues el trabajador podía solicitar atención médico-quirúrgica especializada y de tratamientos de rehabilitación, "siempre que se disponga de los servicios

$\begin{array}{ll}4 & \text { BOCE, no. } 641,14-10-1959, \text { pp. 13332-13333. } \\ 5 & \text { BOCE, no. } 669,12-07-1960, \text { pp. 13975-13976. } \\ 6 & \text { BOCE, no. 860, 03-12-1964, pp. 18396-18397. } \\ 7 & \text { BOCE, no. } 740,30-06-1962 \text {, pp. } 15574-15575 .\end{array}$ 
correspondientes". Como ocurre en el artículo segundo, para percibir tal protección solo se tenía que estar afiliado a la institución de seguridad social del país de origen. En ambos casos, esta última era la institución que se hace cargo de los costos del servicio.

El artículo cuarto se dedica a la protección en la vejez y la invalidez, más concretamente en el caso de que el trabajador hubiese estado empleado y cotizando en los dos países contratantes, y no reuniese, en ninguno de los dos, el período de cotizaciones suficiente para causar derecho. Para garantizar la prestación se recurría a un método simple: la totalización, en que se computaban los tiempos cotizados en ambos países y se concedía un beneficio, que era el resultado de la suma de las pensiones parciales calculadas por cada institución separadamente, relacionada con el porcentaje de sus prestaciones, el tiempo de cotización, y la edad. La institución responsable del pago sería la última donde hubiese cotizado. El artículo quinto trata el tema de la ratificación; el momento de entrada en vigor, un mes después del canje de los instrumentos de ratificación; la duración del convenio, un año; el modo de renovación, tácitamente de año en año; los tiempos de denuncia del acuerdo, al menos tres meses antes de su vencimiento; y la situación tras la denuncia.

Como se ha indicado para el número de artículos, el acuerdo hispanoperuano contiene algunas diferencias. Así, la cobertura ofrecida en el artículo segundo se amplía a la hospitalización y tratamiento farmacéutico. Es en el artículo cuarto donde se aprecia la mayor diferencia. En primer lugar, a las prestaciones de invalidez y vejez se sumaban las de supervivencia. Además, se legislaba claramente a favor de los beneficiarios, como demuestra el hecho de que,

si la cuantía de la prestación a la que el interesado pueda tener derecho por los períodos del seguro cumplidos en virtud de la legislación de una de las partes fuere superior al total de la que resultare a su favor por aplicación del párrafo anterior [referido a la totalización], dicha institución le concederá un complemento igual a la diferencia. (BOCE, no. 860 18397)

Además, si los pensionados mudaban su residencia "a cualquier otro país", mantendrían "el derecho a la transferencia de la prestación que tengan reconocida" (BOCE, no. 860 18397). Tras este artículo se añade un quinto, que refuerza la idea de que para la totalización se tendrían en cuenta los períodos previos a la entrada en vigor del Convenio. Y lo que en los otros convenios se concretaba en el artículo quinto, en este se desdoblaba. El sexto preveía la 
concertación de Acuerdos administrativos para que "los principios de Seguridad Social" entrasen en vigor en un plazo de seis meses desde la ratificación del acuerdo. El séptimo artículo no concretaba la forma de ratificación del mismo, pues se remite a "las normas jurídicas vigentes", por lo que podemos suponer que se seguiría el mismo procedimiento que en los otros convenios.

Como se aprecia, en las cuestiones tratadas, en la estructura, y en la forma del Convenio, estos acuerdos tienen más que ver con aspectos políticos que con necesidades socioeconómicas. Se trataba de meras declaraciones políticas, en que no se especificaba con detalle cómo se garantizarían los propios acuerdos, ni se definían las instituciones gestoras de los sistemas de seguridad social. Precisamente, esta idea se refuerza con una simple comprobación: en las décadas de los sesenta y setenta en Iberoamérica vivían cerca de un millón de españoles, que se concentraron en seis países: Argentina, con 540.000 españoles; Venezuela, 149.747; Brasil, 115.893; Cuba, 74.026; Uruguay, 44.831; y México, 31.038 (Fernández y Kreienbrink 232). Ninguno de estos países concertó acuerdos en esta etapa con España. Tras esta constatación, queremos aventurar algunas hipótesis que puedan explicar por qué los acuerdos concertados se hicieron con países que contaban con una colonia española mínima.

Camilo Ponce Enríquez fue presidente de Ecuador entre 1956 y 1960, y en su mandato prestó cierta atención a la seguridad social, lo que se demuestra en el hecho de que, en 1958, el tercer Congreso de la OISS tuviese lugar en Quito (Vilar y Pons 5). Del mismo modo, edificó un nuevo espacio para la Caja del Seguro, sede del Instituto Ecuatoriano de Seguridad Social. Este interés por la materia de seguridad social, y de relaciones con la España de Franco, podría tener que ver también con su catolicismo militante, pues este presidente precisamente se destaca por ello tras sesenta años de gobiernos liberales jacobinos (Salvador 494).

En el caso peruano, Belaúnde Terry (1963-1968) fue incluido en la Alianza para el Progreso (Williamson 344). La atención a lo social, característica de este programa, y a las relaciones internacionales, se percibe en la exposición de motivos del Convenio en que, a la conveniencia del principio de equiparación de derechos sociales en materia de seguridad social para los nacionales de ambos países (lo social), suma la reafirmación de "el espíritu de protección recíproca plasmado en el Convenio Multilateral de Quito, del III Congreso de la Organización Iberoamericana de Seguridad Social (OISS), adicionando sus principios en el sentido aconsejado por la recíproca experiencia" (BOCE, no. 860 18396). Recordemos que uno de los acuerdos más destacados en este Congreso fue el reconocimiento recíproco de los derechos sociales en los países que suscribieron el tratado de cooperación (Vilar y Pons 5). El artículo cuarto, 
a que ya hemos hecho referencia, introdujo modificaciones, que se publicitaron explícitamente "a mérito del presente Acuerdo" (BOCE, no. 860 18396). No podemos dejar de resaltar esta muestra de superioridad sobre los otros dos convenios alcanzados por España en Iberoamérica, Paraguay y Ecuador, especialmente con este último. La inferioridad del gobierno ecuatoriano respecto del de Perú se mostraría en que las tendencias integradas en el acuerdo hispanoperuano se habrían acordado en el congreso celebrado en la capital de Ecuador. Y esto tendría sentido, pues José María Velasco Ibarra, en su cuarto mandato presidencial en Ecuador, 1960-1961, proclamó la nulidad del Protocolo de Rio de Janeiro de 1942, con el abierto rechazo de Perú (Salvador 499). ${ }^{8}$ Sostenemos que las necesidades diplomáticas de Perú motivaron la firma este Convenio (más bien declaración política), más que las socioeconómicas (presencia de emigrantes españoles en Perú o de peruanos en España).

\subsection{Convenios de la segunda época, 1966-1974}

En esta segunda etapa, se firmaron cuatro convenios: dos con nuevos Estados, Argentina en 1966 y Brasil en 1970. Los otros dos eran un "convenio complementario" de 1972 al ya suscrito con Paraguay y un "convenio adicional" de 1974 al firmado con Ecuador. Los dos primeros acuerdos responden a cuestiones sociopolíticas de los Estados americanos contratantes, mientras que los dos últimos responden a sus condiciones políticas. En los dos primeros convenios no hay una exposición de motivos, que, esencialmente, es una fórmula política.

La emigración española a Argentina se redujo entre 1959 y 1967 en tal grado, que retornaron más migrantes a España, 60.753, de los que marcharon, 60.560. Ante esta situación, se impulsó una estrategia que motivase la emigración de trabajadores cualificados y favoreciese la reagrupación familiar. Así, en 1960, se firmó un convenio de migración que buscaba el asentamiento en suelo argentino de técnicos españoles y colonos agropecuarios. En esta línea, seis años más tarde se firmó el convenio de seguridad social (Fernández y Kreienbrink 240-241).

Por su parte, Brasil también buscó atraer la emigración española cualificada. Sin embargo, y a pesar de comenzar en el mismo año que Argentina con un convenio de emigración, el convenio sobre seguridad social se alcanzó

8 El protocolo significó la cesión a Perú de territorios fronterizos en disputa, que hasta el momento habían estado en manos de Ecuador (Salvador 463-464). 
en 1969 (Fernández y Kreienbrink 243). La situación económica brasileña en la primera parte de los años sesenta no fue amable para la emigración, pues respondía a las medidas impulsadas por Roberto Campos, cuyo objetivo consistía en que la economía respondiese más al mercado que a las decisiones políticas y planificadoras. Mutatis mutandis, el proceso es muy similar al acontecido en la España de los años sesenta, al menos en sus caracteres externos. Ante las dificultades económicas producidas por un planteamiento corporativista y nacionalista (autárquico en el caso español), se impone en España el Plan de Estabilización y Liberalización de 1959, diseñado y desarrollado por unos denominados tecnócratas, representantes del poder económico, designados por representantes del poder político que no habían sido elegidos democráticamente (la dictadura franquista se instaló tras una cruenta Guerra Civil en 1939 y la Junta Militar se impuso en Brasil en 1964 tras un golpe de Estado contra Goulart) (Williamson 419-423). ${ }^{9}$

El Convenio sobre seguridad social firmado entre España y Argentina ${ }^{10}$ se estructura en cuatro títulos, que agrupan diversos artículos según la temática. En el primer título se encuentran las disposiciones referidas a las legislaciones a las que se aplicará el convenio y a determinar quiénes serían sus beneficiarios. El artículo primero explicitaba las contingencias que se iban a cubrir: invalidez, vejez, supervivencia; maternidad; y accidentes y enfermedades laborales. En el lado español se especificaba que todas las prestaciones afectarían tanto a los trabajadores del régimen general como a los de los diferentes regímenes especiales. En el párrafo segundo se afirma que las modificaciones legales "que completen o modifiquen" las legislaciones indicadas también se aplicarían, aunque el párrafo tercero indica la excepción de las que extienden la cobertura a nuevas categorías profesionales no definidas en el convenio. El artículo segundo materializaba la equiparación "a los trabajadores de ambos Estados, los cuales tendrán los mismos derechos y las mismas obligaciones que los nacionales del Estado contratante en cuyo territorio se encontraren" (BOCE, no. 941 20220). Los artículos tercero y cuarto sirven para aclarar a qué trabajadores no se aplica el convenio: trabajadores que realicen su labor por un periodo no superior a los doce meses, el personal de vuelo de las empresas de transporte aéreo y tripulantes de buque, que, temporalmente, actúen en uno de los países contratantes, cuando su empresa este domiciliada en el otro (art. 3). Los funcionarios de carrera de las

9 Para conocer las pugnas políticas y condicionantes económicas previas al Plan de Estabilización y Liberalización en España, así como las primeras consecuencias de su aplicación remitimos a Barciela et al. (178-195). BOCE, no. 941, 09-12-1966, pp. 20219-20224. 
representaciones consulares y diplomáticas, así como los demás funcionarios, empleados y trabajadores al servicio de tales representaciones quedaban ligadas a la legislación del Estado al cual servían (art. 4). El artículo quinto garantizaba la conservación de los derechos adquiridos en las legislaciones indicadas, salvo la maternidad, al trasladarse al otro Estado. También se garantizaba la protección al trasladarse a un tercer Estado "en las mismas condiciones que el Estado que otorga la prestación conceda a sus nacionales residentes en dicho tercer Estado" (BOCE, no. 941 20221).

El título segundo, relativo a las disposiciones especiales, trata el modo en que se calcularán las pensiones. Esencialmente cómo se llevaría a cabo la totalización, cómo se determinaría cuánto había que pagar al trabajador y qué institución habría de hacerlo, en el supuesto de que hubiese cotizado en ambos países. El título tercero, disposiciones varias, explicaba qué autoridades eran las competentes en la aplicación de la materia y cómo se tenían que relacionar. Así, lo más destacado de este título considera que las autoridades competentes máximas son "los Ministros o Secretarios de Estado bajo cuya competencia se encuentren los regímenes enumerados en el artículo 1." " (art. 11.), y la capacidad de "las autoridades diplomáticas y consulares de los dos Estados contratantes" para "representar, sin mandato especial, a los nacionales de su propio Estado, ante las autoridades competentes y ante las entidades gestoras en materia de seguridad social del otro Estado" (art. 18). El cuarto y último título, disposiciones transitorias, regulaba in extenso, con siete párrafos, "el Mutualismo Laboral" en España. Los artículos vigésimo segundo y vigésimo tercero tratan la duración del convenio, tres años a partir de su entrada en vigor, y el modo y lugar de ratificación del mismo, así como su denuncia (art. 22) indicando, finalmente, que "las modalidades de aplicación del presente Convenio serán objeto de un Acuerdo Administrativo" (art. 23.3.).

De este convenio queremos resaltar dos cosas: primera, dos contingencias esenciales no son cubiertas, el desempleo y la enfermedad. En el caso del desempleo se entiende, pues la prestación contra el mismo no se aprobó en Argentina hasta un año después, en 1967, sin embargo, el seguro de enfermedad sí estaba establecido en ambos países (Vilar y Pons 7), aunque no se reguló. Segunda, y en relación con el artículo vigésimo primero, se muestra el cambio estructural en el sistema de seguridad social español de 1963. Hasta esa fecha existieron en España dos subsistemas de seguridad social, el organizado en torno a los seguros sociales con prestaciones unitarias, basado en la idea de generalidad y de "previsión social proletarizada"; y el Mutualismo Laboral, en que las prestaciones eran diferenciales en función de la renta en activo, no siendo además proletarizada, pues incluía a los cuadros 
profesionales superiores (Libro 165). Como destaca la investigación, el Mutualismo Laboral era un sistema complementario a los seguros sociales. Tanto es así que los trabajadores con derecho a prestación de vejez en el Mutualismo cobraban una pensión de 250 ptas. mensuales de los seguros sociales, ascendiendo a 400 ptas. si no tenían derecho al Mutualismo Laboral (González 812).

Esta doble estructura se percibe en el primer artículo, en que se indica que en España las prestaciones relativas a invalidez, vejez y supervivencia se otorgaran "Del régimen general. [...] y Del Mutualismo Laboral". También se muestra la situación diferencial, incluso dentro del propio Mutualismo, pues se mencionan "los Estatutos de la Mutualidad Laboral en que por la profesión respectiva estuvieran afiliados" (art. 21. 2/b). El propio convenio recordaba que esta situación era provisional "hasta la fecha de aplicación en España del Régimen General de la Seguridad Social que regula el texto articulado de la Ley 193/1963" (art. 21.1).

El convenio con Brasi1 ${ }^{11}$ se firmó en 1969, en un momento en que la estructura del sistema de seguridad social español ya había adoptado su forma última durante el franquismo. La estructura del convenio es prácticamente idéntica al caso Argentino, aunque sin el título cuarto, por lo que solo comentaremos los cambios más destacados.

En el artículo primero se mencionan las prestaciones a que se aplicará el convenio: asistencia médico-sanitaria y subsidio de incapacidad laboral transitoria; vejez; invalidez; muerte; natalidad; y, exclusivamente en el caso del "Sistema General de Previsión Social" de Brasil, el tiempo de servicio. A diferencia del caso argentino, este convenio sí se aplicaría a las disposiciones que extendiesen los regímenes existentes a nuevas categorías profesionales o creasen nuevas ramas de seguridad social. El artículo quinto añade un segundo párrafo garantista para con los trabajadores según el cual los "que tengan en suspenso las prestaciones correspondientes a los derechos relacionados en el artículo $1 .^{\circ}$, por el hecho de haberse trasladado al territorio del otro Estado contratante, podrán readquirirlas a petición propia, en virtud del presente Convenio" (BOCE, no. 1.091 26623). Luego desarrollaba unas condiciones para la adquisición. Además, se añadía un sexto artículo, relativo a la asistencia sanitaria de los viajeros no migrantes. En el primer párrafo, se trataba el tema del trabajador que viajase de un país contratante a otro en vacaciones ("con ocasión de permisos remunerados"), a quien se garantizaba la asistencia sanitaria siempre que estuviese "afiliado a la 
Previsión Social de un Estado contratante" y a "las personas que dependan del referido trabajador que lo acompañen en su desplazamiento" (BOCE, no. 1.091 26.623). A estos se refería el segundo párrafo, pues se les garantizaba asistencia sanitaria en su país de origen durante doce meses tras afiliarse el trabajador emigrante al sistema de seguridad social del otro país contratante. El párrafo cuarto indicaba que debía correr con todos estos costos la entidad en la que estuviese afiliado el trabajador.

En el artículo séptimo, que en el argentino era el sexto, se añaden dos párrafos. El cuarto regulaba la pensión de vejez o por tiempo de servicio. En él se indicaba que la concesión de tal pensión correspondería a la última institución en que estuviese afiliado el trabajador, siempre que hubiese cotizado un mínimo de cinco años y cumplido los sesenta años. El quinto garantizaba a los trabajadores migrantes los subsidios de incapacidad laboral transitoria y la asignación por natalidad en el país de acogida, siempre que en el país de origen tuviesen completado el período de carencia para causar derecho. En el supuesto de que no fuese así, se reconocía el derecho si la suma de los periodos cotizados en ambos países era suficiente para completar el período de carencia. Como última diferenciación con el caso argentino, el último artículo, el vigésimo cuarto, sustituía los artículos del cuarenta y cuatro al cincuenta y uno, ambos incluidos, del acuerdo de migración suscrito entre ambos países en Madrid en 27 de diciembre de 1960, "quedando a salvo los posibles derechos adquiridos al amparo de los mismos" (BOCE, no. 1.09126 .626 ).

De este convenio también queremos destacar dos cuestiones: primera, potenciaba mucho más la emigración y la reagrupación familiar que el argentino. En primer lugar, garantizaba la prestación y cobertura en la enfermedad (no solo en la maternidad), y, en segundo lugar, empujaba a la reagrupación familiar. Solo así se explica que la familia dejada en el país de origen tuviese una cobertura sanitaria limitada a un año si el trabajador emigraba. La única forma de que la familia fuese protegida por la asistencia sanitaria de la seguridad social era emigrar junto al trabajador. La segunda cuestión tiene que ver con dos conceptos. Hasta este Convenio solo nos habíamos encontrado con el concepto seguridad social, término anglosajón (acuñado por Franklin D. Roosevelt y popularizado por William Beveridge). ${ }^{12}$ Este concepto se impuso en el concierto internacional tras el fin de la Segunda Guerra Mundial para nombrar los sistemas estatales

12 Simón Bolívar utilizó el término ante el Congreso de Angostura, pero sin el sentido con que lo popularizaron Roosevelt y Beveridge (Rubio 240). 
integrados de seguros y servicios sociales, así como la asistencia social y, en casos concretos, la sanidad pública (Ritter 30-32). Aunque, según este sentido, el régimen franquista no podía ser considerado como tal, utilizó el término de una forma propagandística (González 647). Es por ello que no le costó adoptarle para firmar convenios con otros Estados. Sin embargo, en este documento, sobre todo en relación con Brasil, también se utiliza el concepto de previsión social, término castellano, centrado en los seguros sociales enfocados al trabajador afiliado, equivalente a previdência social en lengua portuguesa.

Los dos convenios siguientes no responden anecesidades socioeconómicas. Muestra de ello son sus respectivas exposiciones de motivos. Aunque la redacción no es exactamente idéntica, podemos tomar el convenio hispano-paraguayo como ejemplo. Los dos gobiernos sudamericanos acordaron el convenio con España pues estaban "animados del deseo de mejorar las relaciones existentes entre ambos en el campo de la Seguridad Social", "teniendo en cuenta la evolución legislativa experimentada en dicha materia" (BOCE, no. 1.215 29.524).

El convenio hispano-paraguayo ${ }^{13}$ se estructuraba de forma semejante a los otros, con cuatro títulos, aunque con variaciones importantes. En el título primero, disposiciones generales, antes de entrar en materia se definen algunas expresiones, ${ }^{14}$ gracias a las cuales sabemos que las autoridades competentes eran el Ministerio de Trabajo en España y los Ministerios de Justicia y Trabajo, y de Salud Pública y Bienestar Social en Paraguay. En el artículo segundo es donde se incluyen las prestaciones que se iban a proteger: asistencia sanitaria e incapacidad laboral transitoria; invalidez provisional y permanente; vejez; muerte y supervivencia; accidentes de trabajo y enfermedades profesionales; y prestaciones familiares. También se definen los colectivos a cubrir a través de la enumeración de los distintos regímenes de los sistemas de la seguridad social. En el caso español, al régimen general se suman el de los trabajadores agrícolas asalariados; trabajadores del mar; trabajadores ferroviarios; trabajadores de la minería del carbón; y servicio doméstico. En el caso paraguayo, la protección es para los trabajadores asimilados al régimen general y a los regímenes especiales de trabajadores ferroviarios y empleados bancarios. En lo referido a las posibles modificaciones legislativas, se trata como el convenio argentino, es decir, el convenio se aplicaría a los regímenes y seguros que complementen, refunden o amplíen los beneficios de las disposiciones legales; pero no de las que

13 BOCE, no. 1.215, 22-06-1972, pp. 29523-29529.

14 Territorio, nacional, legislación o disposiciones legales, autoridad competente, organismo, organismo competente, organismo del lugar de residencia, período de cotización, período equivalente y prestaciones económicas. 
establezcan nuevos seguros o amplíen la acción protectora con nuevas categorías de trabajadores. La misma semejanza se da en el caso de los trabajadores que se desplacen a trabajar temporalmente de un país contratante a otro, los tripulantes de barcos y los de compañías de transporte aéreo (art. 4), así como los cuerpos de las representaciones diplomáticas y consulares (art. 5).

El título segundo, disposiciones especiales, aporta una novedad estructural, pues los artículos se agrupan en capítulos, referidos cada uno a contingencias concretas: enfermedad, maternidad y subsidio; vejez, invalidez y supervivencia; accidentes de trabajo y enfermedades profesionales; y prestaciones familiares. En mayor o menor medida, las ideas de la protección son las mismas (en la enfermedad y en la vejez) que en el convenio anterior, por lo que, aunque la redacción de este sea más amplia y precisa, solo comentaremos los cambios más importantes. Así, la protección por enfermedad ofrecida al trabajador en el anterior convenio se extiende a sus familiares, $\mathrm{y}$ a las personas que ya no fueran trabajadores sino pensionistas de uno de los sistemas de seguridad social de los países contratantes, siempre que entre sus derechos reconocidos se encontrase la asistencia sanitaria (art. 7.3.). El artículo octavo es una novedad por cuanto regula las prestaciones por defunción, en que, si no se cumplen los requisitos de cotización en el país de trabajo, se utilizarían los períodos en el otro, a fin de causar derecho. En el capítulo de las prestaciones por vejez, invalidez y supervivencia sí hay novedades. Se tenían en cuenta los periodos cotizados en terceros países para la totalización de los períodos en ambos países contratantes para el cálculo de las prestaciones de vejez y supervivencia, siempre que entre Paraguay y España "se hayan convenido normas similares con estos terceros países" (art. 11). En el caso de los accidentes laborales y enfermedades profesionales; así como las prestaciones familiares, el acuerdo constituía una novedad. Hay que indicar que se tendía a la máxima protección normativa para emigrante. El artículo décimo tercero garantizaba que se tomaran en consideración los accidentes de trabajo y enfermedades profesionales acontecidos en el otro país como si se hubiesen producido en el país en que estuviesen trabajando en el momento. El artículo décimo cuarto mantiene el derecho en el país de destino a la prestación sanitaria como consecuencia de accidentes o enfermedades profesionales. En las prestaciones familiares se reconoce la paridad entre los trabajadores, y en el supuesto de que se exija alguna carencia, se totalizarían los períodos cotizados en ambos países (art. 15).

En el título tercero, disposiciones diversas, se trata el tema de las autoridades competentes y de los organismos de enlace. En otros convenios estos últimos no se especifican, pero en este sí. En el caso español lo sería el Instituto Nacional de Previsión y en el caso paraguayo el Instituto de Previsión Social (art. 
16). El título cuarto, disposiciones transitorias y finales, determina los modos de ratificación, y las consecuencias de su aplicación respecto al anterior convenio.

El último convenio sobre seguridad social firmado por el franquismo con Iberoamérica fue el convenio hispano-ecuatoriano de 1974. En este caso, las necesidades políticas del Presidente de Ecuador tienen gran peso. Este era el general Guillermo Rodríguez Lara que, junto con el Consejo Militar de Gobierno el 15 de febrero de 1972, al que encabezaba, dio un golpe contra José María Velasco Ibarra, quien en junio de 1970 había suprimido el Congreso y asumido plenos poderes. Guillermo Rodríguez pronto se hizo nombrar como Presidente de la República. Se ganó la simpatía de los países socialistas, por cuanto su plan de gobierno tenía claras influencias de la izquierda progresista peruana representada por Juan Velasco Alvarado. También Ecuador entró a formar parte de la OPEP, por el descubrimiento de pozos petrolíferos y una producción diaria de 200.000 barriles. Precisamente, esta pertenencia motivó un viaje de Guillermo Rodríguez en que asistió a una cumbre de los jefes de Estado de los países miembros de la OPEP en Argel. En el viaje visitó países socialistas, como Rumanía, y países iberoamericanos como Venezuela y España (pues paró en las islas Canarias). En palabras de Jorge Salvador, "viaje sin real trascendencia para la nación y más bien rodeado de pintoresco sensacionalismo inconsistente", pero que mostró la "tranquilidad y estabilidad en las relaciones internacionales" (Salvador 524-528). El convenio se encuadraría dentro de esta política.

Este convenio ${ }^{15}$ se articuló en tres grandes títulos, precedidos de una exposición de motivos, que ya hemos comentado. En el primero, no hay una lista de expresiones definida (a diferencia del convenio hispano-paraguayo) y comienza enumerando los beneficios que se otorgan en cada país. En España las prestaciones reconocidas son: asistencia sanitaria e incapacidad laboral transitoria; invalidez provisional y permanente; vejez; muerte y supervivencia; y accidentes de trabajo y enfermedades profesionales. Además, aparte del régimen general, se incluían los regímenes especiales: régimen agrario; trabajadores del mar; trabajadores por cuenta propia; trabajadores ferroviarios; trabajadores de las minas de carbón; empleados del hogar; representantes de comercio; escritores de libros; artistas; estudiantes; y toreros. En Ecuador se garantizaba la afiliación a los siguientes seguros: enfermedad y maternidad; subsidio de enfermedad; de Invalidez; de Vejez; de Muerte; y Riesgos del Trabajo; además de las legislaciones especiales del Seguro de Artesano y del Seguro de los 
Trabajadores Domésticos. En el artículo segundo se garantizaba la cobertura a los trabajadores, derechohabientes y familiares "en iguales condiciones que los nacionales de cada uno de los dos países" (BOCE no. 1.362 33222). Los artículos tercero y cuarto trataban, respectivamente, las consabidas situaciones especiales de trabajadores temporalmente en un país, de compañías aéreas y de tripulaciones de barcos, así como los funcionarios y trabajadores de las representaciones diplomáticas y consulares en el país de destino. El artículo quinto expone las Autoridades Competentes: en España, "el Excelentísimo Señor Ministro de Trabajo"; en Ecuador, "el Excelentísimo Señor Ministro de Trabajo y Bienestar Social, Presidente del Consejo Superior del Instituto Ecuatoriano de Seguridad Social" (BOCE, no. 1.362 33223).

El título segundo, disposiciones especiales, organiza los artículos en capítulos que tratan unas contingencias concretas, si bien deja dos capítulos desgajados al principio que sirven de marco general. El artículo sexto garantiza a los trabajadores, que percibiesen una prestación económica en su país de origen y que migrasen al otro país contratante, que continuarían disfrutándola a cargo de la entidad del país primero. El artículo séptimo trata el tema de la totalización, como en otros convenios, si bien complementándolo con lo anterior, pues "no se opondrá a la indicada totalización la circunstancia de que los períodos de cotización, a que la misma afecta, hayan dado lugar al otorgamiento de alguna prestación" (art. 7. b). El capítulo primero se dedica a la enfermedad, que es tratada como en el anterior convenio. El capítulo segundo se dedica a las prestaciones por vejez, invalidez derivada de enfermedad común o accidente laboral y supervivencia. El artículo décimo se dedica a la totalización, muy semejante a la del anterior convenio. El undécimo, indica que, para la concesión de las pensiones de supervivencia, la determinación de los familiares del trabajador o pensionista que tengan derecho a la misma, cuando una de las partes los haya determinado, la encargada de otorgarlas debe tenerla en cuenta, si bien aplicando su legislación. El artículo décimo segundo expone cómo se ha de actuar en el caso de que "si para reconocer derecho a pensión de vejez la legislación de cada una de las Partes Contratantes exigiera mínimos diferentes" (BOCE, no. 1.362 33225). Para controlar la prestación a que tenía derecho el trabajador, se aplicarían coeficientes reductores en el país que tuviese establecida un límite de edad superior en el porcentaje de la pensión a su cargo. Evidentemente, esto servía para la totalización. El artículo décimo cuarto, referido a los nacionales de uno de los Estados en un tercer país, lo trata de igual forma que el convenio hispano-paraguayo. El artículo décimo quinto trata la invalidez, señalando que "la calificación y determinación del grado de invalidez del solicitante corresponderá a la Institución que haya de otorgar 
la pensión" (BOCE, no. 1362 33.225). El capítulo tercero trata el asunto de las prestaciones por accidente de trabajo o enfermedad profesional. En los artículos se garantiza el reconocimiento de los accidentes y enfermedades profesionales en uno de los países que sirviesen para causar derecho en el otro, y los controles a los afectados por una de estas contingencias, en las condiciones que indicase la entidad obligada, aunque debe aplicarlo la entidad del otro país (arts. 16-17). El capítulo cuarto se dedica a la muerte (subsidio por defunción), que se garantizaba con dos requisitos: estar afiliado a la institución de seguridad social del país de empleo, y cumplir los requisitos, aunque de no cumplirse, se totalizarían los períodos en ambos países. El título tercero, disposiciones finales, trata a las autoridades competentes y el modo de ratificación y entrada en vigor del nuevo convenio, en que se reconocía el mantenimiento de los derechos adquiridos en base al anterior, indicándose que la duración será la misma que en el anterior.

Estos dos convenios son muy interesantes por cuanto nos permiten conocer el grado de desarrollo de los sistemas de seguridad social. Salvo las contingencias de desempleo, al menos en su normativa, todos los sistemas cubrirían el resto de contingencias que se esperan de los sistemas de seguridad social desarrollados. La excepción de la protección frente a la contingencia de desempleo podría deberse a que los Estados iberoamericano no la reconocían, o a que el exceso de mano de obra no cualificada en estos Estados era una carga demasiado grande como para añadir la de los posibles migrantes españoles parados. También nos permite acercarnos a su estructura institucional, pues en estos países, la seguridad social se ligaba al Ministerio de Trabajo (de Trabajo en España, de Justicia y Trabajo en Paraguay, y de Trabajo y Bienestar Social en Ecuador), y era gestionada por Institutos (Nacional de Previsión en España, de Previsión Social en Paraguay, y Ecuatoriano de Seguridad Social en Ecuador). Precisamente, la ligazón al departamento dedicado al Trabajo nos habla de un modelo profesionalista de seguridad social, en que la protección se otorgaba a la población a través de los trabajadores afiliados al sistema de seguridad social, y por medio de ellos a sus derechohabientes, esencialmente sus familias. Esta concepción solo es matizada por el caso paraguayo, en que la existencia de un Ministerio de Salud Pública y Bienestar Social parece mostrar una tendencia a la universalización de la sanidad. También nos hablan de diversas formas en la protección a la población trabajadora, basada en las categorías profesionales. Así, junto al régimen general, el franquismo ofrecía protección a la mayoría de la población trabajadora, por medio de regímenes especiales, lo que muestra la supervivencia de cierto corporativismo, a pesar de los cambios en la estructura económica establecida por el Plan de Estabilización y Liberalización (De la 
Calle 300-301). ${ }^{16}$ En el caso paraguayo, dos serían los regímenes especiales, el de los trabajadores ferroviarios y trabajadores bancarios. En Ecuador, las características especiales darían en dos seguros distintos al general, el de artesanos y el de trabajadores domésticos. O bien estos dos últimos países apenas tenían un componente corporativista en su sistema y por ello incluían a la inmensa mayoría de trabajadores en el régimen general, o bien aún no habían establecido su cobertura a toda la población trabajadora. También hay que resaltar una característica aplicable a todos los convenios de la segunda fase, que denota, asimismo, una característica de la estructura económica de todos los países contratantes: su fuerte emigración al extranjero, a terceros países, de ahí que se incluyesen cláusulas relativas a la equiparación de los propios nacionales con los nacionales de los otros países contratantes, en países ajenos a los convenios.

Los técnicos redactores del Libro Blanco de la Seguridad Social de 1977, que estudiaban los defectos y proponían las mejoras que podían introducir al sistema para "la Seguridad Social en una nueva etapa de la vida española" (Libro 7), aducían la falta de rigor técnico y las notables ausencias de estos convenios. Con ellos finalizamos:

Sería aconsejable un replanteamiento en profundidad de los Convenios de Seguridad Social suscritos con los países que forman la comunidad iberoamericana de naciones, modernizando los existentes y extendiendo las negociaciones a otros países con los que aún no se ha concertado convenios de esta índole (Chile, Venezuela, Uruguay y, en su día, Méjico, etc.)" (Libro 392). ${ }^{17}$

16 Esta situación hace referencia a la normativa emanada del régimen franquista, que, como ha demostrado la historiografía referida al tema, no se correspondía con la realidad social, ya que las autoridades franquistas ni le dedicaron el gasto social público necesario para ello ni tuvieron una vocación política plena para hacerlo. Para profundizar en el tema remitimos a los artículos "El mito de la política social franquista: vivienda, educación y seguridad social" de Ortiz Heras y González Madrid y "El franquismo y la construcción del Estado de Bienestar en España: la protección social del Estado (1939-1986)" de González Madrid y Ortiz Heras, quienes sintetizan prácticamente toda la producción historiográfica sobre la seguridad social franquista.

17 La inclusión aquí de Chile, donde la colonia española no era tan numerosa como en el resto de países de la lista (en que sí lo era), responde, (paradójicamente, porque España afrontaba una transición de una dictadura a una democracia), a la necesidad de legitimidad internacional del régimen dictatorial de Augusto Pinochet (admirador de Franco), desplazado del concierto internacional por su golpe contra el régimen democrático anterior (Vázquez 108-109). 


\section{CONCLUSIONES}

Hasta mediados de la década de los sesenta no se dieron las condiciones socioeconómicas que justificasen la firma de convenios de seguridad social entre la España de Franco y los Estados iberoamericanos con los que se acordaron tales convenios. Es por ello que los convenios firmados en la primera mitad de esta década respondían a necesidades políticas. Necesidades, además, que intuimos, y así lo hemos defendido, en estas fechas, eran de los Estados de América. Y esto sería así porque el régimen franquista estaba más interesado en potenciar la emigración hacia Europa, con la que ya tenía unas relaciones diplomáticas cordiales y que le generaba importantes réditos económicos, a diferencia de la situación de las décadas de los cuarenta y cincuenta, cuando había iniciado su estrategia iberoamericana. Es por ello por lo que los convenios acordados en esta época eran poco más que una declaración de intenciones con menos de diez artículos. En la segunda mitad de los sesenta, solo algunos de los convenios sobre seguridad social respondían a cuestiones socioeconómicas, fundamentalmente los de Argentina y Brasil, deseosos de potenciar la inmigración española. A la muerte del dictador, los técnicos que planificaron el sistema de seguridad social español solicitaban la firma de convenios con países en que la colonia española fuese sustanciosa, y que respondiesen, no a criterios políticos, sino técnicos.

Así, del estudio de los convenios podemos obtener importantes informaciones. Por una parte, el grado de desarrollo institucional de los sistemas de seguridad social, en base a las contingencias que se incluían en ellos y los colectivos a quienes se protegía en los acuerdos. Por otra parte, el modo en que se estructuraban los sistemas, así como las concepciones sobre la materia que había en los distintos Estados, en base a las denominaciones de los Ministerios en que se incluía tal estructura, además de sus entidades directamente gestoras, fundamentalmente Institutos. También, de las relaciones con terceros países, no mencionados hasta los convenios del segundo tipo, podemos deducir el carácter o la situación de la economía de los países contratantes, pues todos regulan situaciones de emigración de los propios nacionales, lo que se relaciona con el boom demográfico posterior a la Segunda Guerra Mundial y la imposibilidad de los Estados de absorber la nueva mano de obra no cualificada, precisamente a partir de tales fechas.

Respecto de la protección ofertada, los Estados acordaron la equiparación normativa plena de los inmigrantes con los nacionales en las contingencias reguladas en los convenios, que no tenían que corresponder con todas las ofertadas en cada país a sus nacionales, como hemos visto en el caso argentino. Además, el modo en que se arbitraba la totalización buscaba ser ventajosa para el inmigrante. 
De este modo, en los convenios firmados en la primera fase, se realizaba tal equiparación en las prestaciones relacionadas con la asistencia sanitaria y con la vejez. En los convenios de segunda fase, la protección se amplió a una gama mayor de contingencias. En este caso, cada convenio, y dependiendo del grado de desarrollo de los sistemas de seguridad social de sus Estados contratantes, incluía la protección frente a unas contingencias u otras. La única excepción fue la contingencia de desempleo, no regulada en ninguno de los convenios.

Como hemos planteado desde el principio, este estudio es una primera aproximación al tema. La lista de temas por estudiar es muy amplia: percepción de los trabajadores emigrantes referida a la protección acordada en los convenios; la comprobación de que tal percepción respondía a la realidad; análisis pormenorizado de las razones que motivaron la firma de estos acuerdos, de tal forma que se puedan ampliar, matizar o refutar las aquí propuestas; y el establecimiento de comparaciones entre los diversos Estados en cuanto a los sistemas de seguridad social. Los métodos y las fuentes para el estudio son variadas, y aquí solo reseñaremos algunas: la historia oral para el conocimiento de las percepciones; el estudio de la documentación generada por los Ministerios de Asuntos Exteriores para conocer las motivaciones precisas del acuerdo de los convenios, y los Ministerios e Institutos que gestionaban los sistemas de seguridad social para conocer el funcionamiento real de dichos sistemas; y expurgos de la bibliografía existente sobre los diversos sistemas para establecer las comparaciones; entre otros. En cualquier caso, esta lista no agota las muchas posibilidades, y sirve más bien de guante que arrojamos con el deseo de que alguien se aventure al estudio que este trabajo solo ha esbozado.

\section{REFERENCIAS BIBLIOGRÁFICAS}

Alonso Olea, Manuel. Instituciones de Seguridad Social. Instituto de Estudios Políticos, 1959.

Álvarez Rosete, Arturo. Social welfare policies in non-democratic regimes: the developtment of social insurance schemes in Franco's Spain (19361950). Tesis doctoral. Universidad de Nottingham, 2005. En línea. nottingham.ac.uk/12084/

Barciela, Carlos, et al. La España de Franco (1939-1975). Economía. Editorial Síntesis, 2001.

Borrajo Dacruz, Efrén. "El trabajador extranjero en el derecho laboral de España. Notas para un estudio". Cuadernos de Política Social, no. 38, 1958, pp. 7-58. dialnet.unirioja.es/servlet/articulo?codigo $=2496439$ 
De la Calle Velasco, $\mathrm{M}^{\mathrm{a}}$ Dolores. "El sinuoso camino de la política social española". Historia contemporánea, no. 17, 1997, pp. 287-308. www. ehu.eus/ojs/index.php/HC/article/view/19949/17806

"Convenios de seguridad social y legislación de aplicación". Economía y Sociología del Trabajo, no. 8-9, 1990, pp. 148-151.

Fernández Vicente, María José, y Axel Kreienbrink. "Las relaciones del IEE con los países de ultramar". Historia del Instituto Español de Emigración. La política migratoria exterior de España y el IEE del Franquismo a la Transición, Luis M Calvo Salgado, et al., Ministerio de Trabajo e Inmigración, 2009, pp. 231-251.

González Madrid, Damián A., y Manuel Ortiz Heras. "El franquismo y la construcción del Estado de Bienestar en España: la protección social del Estado (1939-1986)". Pasado y Memoria. Revista de Historia Contemporánea, no. 17, 2018, pp. 361-388. https://doi.org/10.14198/pasado2018.17.13

González Murillo, Pedro. La política social franquista: el Ministerio de José Antonio Girón de Velasco (1941-1957). Tesis Doctoral. Universidad Complutense de Madrid, 1998. En línea. ucm.es/26326/1/T22382.pdf

Libro blanco de la Seguridad Social. $2^{\circ}$ Ed. Ministerio de Trabajo, 1977.

Ortiz Heras, Manuel, y Damián A. González Madrid. "El mito de la política social franquista: vivienda, educación y seguridad social”. ¿Qué sabemos del franquismo? Estudios para comprender la dictadura de Franco, coordinador Manuel Ortiz Heras, Comares, Granada, 2018, pp. 43-67. https://doi.org/10.5209/chco.66138

Ritter, Gerhard A. El estado social, su origen y desarrollo en una comparación internacional. Ministerio de Trabajo y Seguridad Social, 1991.

Rubio Lara, Ma Josefa. La formación del Estado social. Madrid: Ministerio de Trabajo y Seguridad Social, 1991.

Salvador Lara, Jorge. Breve historia contemporánea de Ecuador. Fondo de Cultura Económica, 1994.

Vázquez Feria, Pedro. "Del Mediterráneo al cono sur: las transiciones a la democracia de Chile, Portugal y España en perspectiva comparada". Revista de Historia Social y de las Mentalidades, vol. 22, no. 2, 2018, pp. 103-135. www.revistas.usach.cl/ojs/index.php/historiasocial/ article/view/3644/26002940

Vilar Rodríguez, Margarita, y Jerònia Pons Pons. "La extensión del seguro de salud en Iberoamérica: una estrategia de política exterior del franquismo 
en la inmediata posguerra (1942-1959)". Asclepio. Revista de Historia de la Medicina y de la Ciencia, no. 67, enero-junio 2015, pp. 1-14. https://doi.org/10.3989/asclepio.2015.13

Williamson, Edwin. Historia de América Latina. Fondo de Cultura Económica, 2013.

\section{Fuentes}

BOE, no. 335, 01-12-1955 p. 7255.

BOCE, no. 641, 14-10-1959, pp. 13332-13333.

BOCE, no. 669, 12-07-1960, pp. 13975-13976.

BOCE, no. 740, 30-06-1962, pp. 15574-15575.

BOCE, no. 860, 03-12-1964, pp. 18396-18397.

BOCE, no. 941, 09-12-1966, pp. 20219-20224.

BOCE, no. 1.091, 05-03-1970, pp. 26621-26626.

BOCE, no. 1.215, 22-06-1972, pp. 29523-29529.

BOCE, no. 1.362, 06-07-1974, pp. 33221-33227. 